\title{
Graphene-Based Adaptive Thermal Camouflage
}

Omer Salihoglu, ${ }^{\dagger}$ Hasan Burkay Uzlu, ${ }^{\dagger}$ Ozan Yakar, ${ }^{\dagger}$ Shahnaz Aas, ${ }^{\dagger}$ Osman Balci, ${ }^{\dagger}$ Nurbek Kakenov, ${ }^{\dagger}$

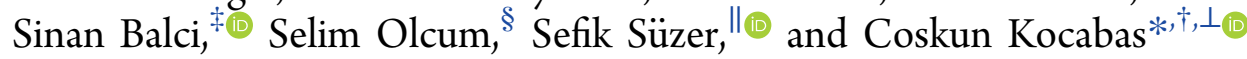

${ }^{\dagger}$ Department of Physics, Bilkent University, 06800, Ankara Turkey

${ }^{\ddagger}$ Department of Photonics, Izmir Institute of Technology, 35430 Izmir, Turkey

${ }^{\S}$ Department of Biological Engineering, Massachusetts Institute of Technology Cambridge Massachusetts 02139-4307, United

States,

"Department of Chemistry, Bilkent University, 06800, Ankara Turkey

${ }^{\perp}$ School of Materials and National Graphene Institute, University of Manchester, Oxford Road, Manchester, M13 9PL, United

Kingdom

\section{Supporting Information}

ABSTRACT: In nature, adaptive coloration has been effectively utilized for concealment and signaling. Various biological mechanisms have evolved to tune the reflectivity for visible and ultraviolet light. These examples inspire many artificial systems for mimicking adaptive coloration to match the visual appearance to their surroundings. Thermal camouflage, however, has been an outstanding challenge which requires an ability to control the emitted thermal radiation from the surface. Here we report a new class of
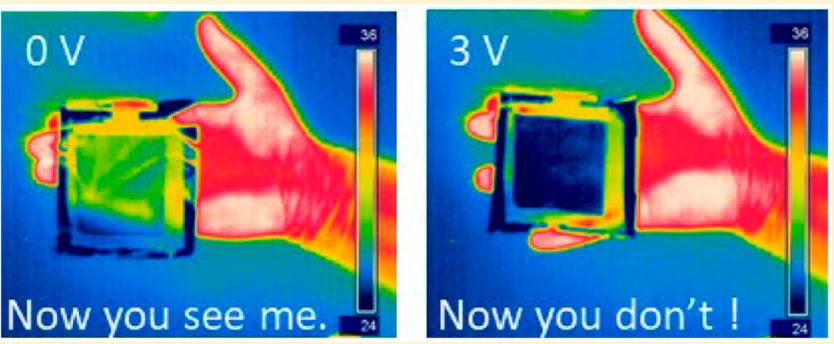
active thermal surfaces capable of efficient real-time electricalcontrol of thermal emission over the full infrared (IR) spectrum without changing the temperature of the surface. Our approach relies on electro-modulation of IR absorptivity and emissivity of multilayer graphene via reversible intercalation of nonvolatile ionic liquids. The demonstrated devices are light $\left(30 \mathrm{~g} / \mathrm{m}^{2}\right)$, thin $(<50 \mu \mathrm{m})$, and ultraflexible, which can conformably coat their environment. In addition, by combining active thermal surfaces with a feedback mechanism, we demonstrate realization of an adaptive thermal camouflage system which can reconfigure its thermal appearance and blend itself with the varying thermal background in a few seconds. Furthermore, we show that these devices can disguise hot objects as cold and cold ones as hot in a thermal imaging system. We anticipate that, the electrical control of thermal radiation would impact on a variety of new technologies ranging from adaptive IR optics to heat management for outer space applications.

KEYWORDS: Graphene optoelectronics, variable emissivity, electrolyte gating, thermal camouflage, thermal emission, multilayer graphene, reconfigurable surface, heat management, IR optics

$\mathrm{T}$ he ability to control thermal radiation from a hot object has both scientific ${ }^{1-5}$ and technological importance. ${ }^{2,6-8}$ The radiated thermal energy per unit area from a hot surface is characterized by the Stefan-Boltzmann law, $P=\varepsilon \sigma T^{4}$ where $\varepsilon$ is the emissivity of the surface, $\sigma$ is the Stefan-Boltzmann constant, and $T$ is the temperature of the surface. The emissivity is the only material-dependent parameter that varies with the wavelength and temperature. At thermodynamic equilibrium, Kirchhoff's radiation law connects the wavelengthspecific thermal emissivity with the optical absorption of the surface as $\varepsilon(T, \lambda)=\alpha(T, \lambda)$. One can engineer the thermal radiation by coating the surface with photonic crystals ${ }^{5,9-11}$ or plasmonic structures. ${ }^{12}$ The dynamic control of thermal radiation, however, requires the ability to alter optical absorption via electrical means. Phase change materials, ${ }^{13-16}$ quantum wells, ${ }^{17}$ electrochromic dyes, ${ }^{18}$ ferroelectric materials, ${ }^{19}$ or plasmonic resonators ${ }^{12,20,21}$ have all been investigated for tunable infrared emission. These research efforts on dynamic control of thermal radiation have encountered various problems such as, low tunability, ${ }^{19,20,22}$ narrow spectral window, ${ }^{17}$ slow response speed, ${ }^{18}$ and rigid substrates. ${ }^{17}$ Electrochromic materials have been the most promising one, ${ }^{23-25}$ however, the requirement of a top metallic contact layer and volatile electrolytes limit their performance (see the benchmarking in Table S1). These challenges have been hindering the realization of adaptive thermal camouflage systems.

Graphene provides new perspectives to control electromagnetic radiation in a very broad spectral range from visible to microwave frequencies. ${ }^{26-33}$ Optical absorption of graphene can be tuned by electrostatic gating owing to the Pauli blocking. ${ }^{34,35}$ Although optical response of graphene has been studied extensively, the use of graphene for dynamic control of

Received: April 29, 2018

Revised: June 5, 2018

Published: June 27, 2018 

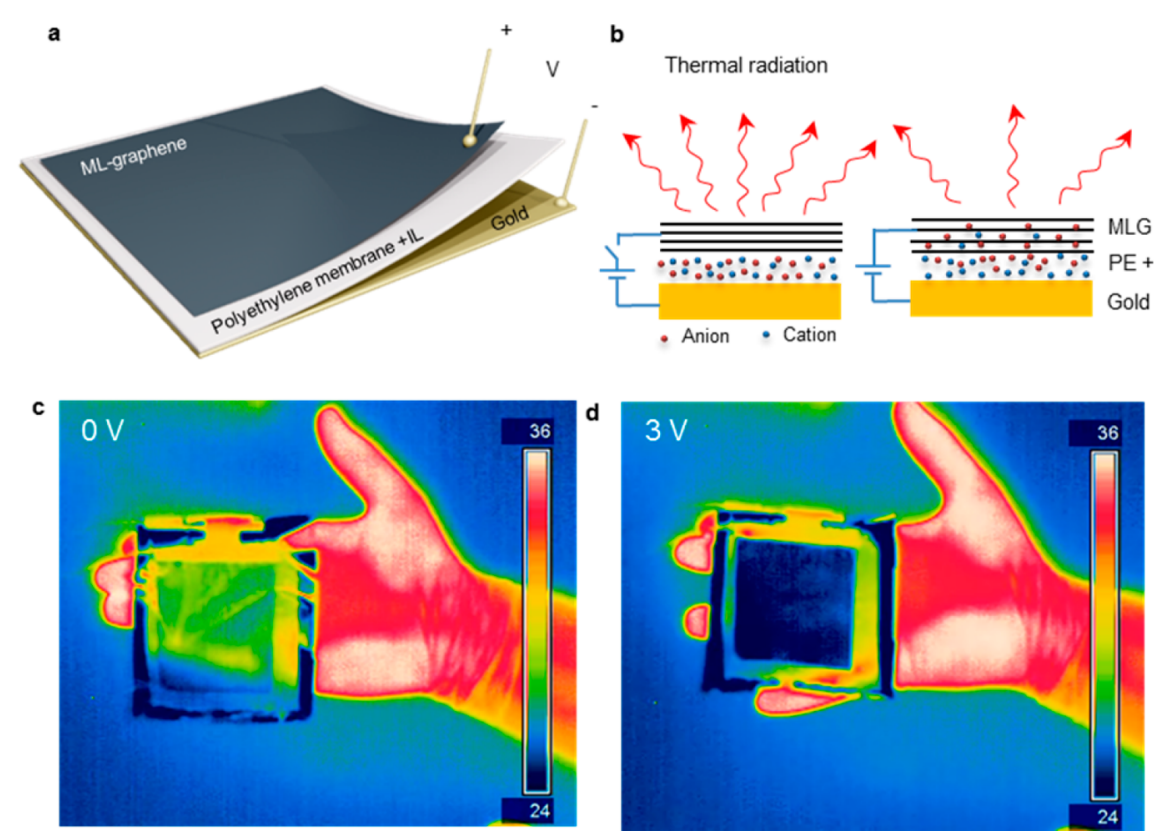

Figure 1. Active thermal surfaces. (a) Schematic drawing of the active thermal surface consisting of a multilayer-graphene electrode, a porous polyethylene membrane soaked with a RTIL, and a back gold-electrode coated on heat resistive nylon. (b) Schematic representation of the working principle of the active thermal surface. The emissivity of the surface is suppressed by intercalation of anions into the graphene layers. (c,d) Thermal camera images of the device placed on the author's hand under the voltage bias of 0 and $3 \mathrm{~V}$, respectively.

thermal radiation has remained unexplored because of the small optical absorption $(<2 \%)$ in mid-IR region. ${ }^{36}$ In this work, we developed a new class of active thermal surfaces using multilayer graphene, which yields significant tunable optical absorption in IR region. Because thermal radiation originates from the very top surface, top-gating or electrolyte gating schemes are not suitable for the control of thermal radiation. These gating methods generate either buried graphene surfaces or low electrostatic doping, ${ }^{34,37}$ which yields negligible IR modulation. None of the previously reported graphene devices by our group and others are suitable for dynamic control of thermal radiation. Therefore, we introduce a new gating scheme using an inverse device structure, which leads intercalation of a nonvolatile ionic liquid into graphene layers from the porous substrate. The inverse device configuration yields an uncovered graphene surface with tunable charge density and Fermi energy. Figure 1a shows the schematic of the active thermal surface consisting of a multilayer-graphene electrode on a porous polyethylene (PE) membrane and a back gold-electrode. We synthesized multilayer-graphene on nickel foils using a chemical vapor deposition method and then transferred them on PE membrane, which is IR transparent and can hold the electrolyte (room-temperature ionic liquid, RTIL).

The thermal radiation emitted by the device mainly originates from the top graphene electrode because the emissivity of gold-coated substrate is very low $(<0.01)$ due to its highly reflective nature and IR transparency of the PE membrane. The gold electrode also prevents transmission of the background thermal radiation. Figure $1 \mathrm{~b}$ illustrates the working principle of the active thermal surface. Under a voltage bias, the ionic liquid intercalates into the graphene layers and dopes them. As a result of doping, the charge density on graphene increases and Fermi-level shifts to higher energies, which suppress the IR absorption and thus the emissivity of the graphene electrode. ${ }^{35}$ Figure $1 \mathrm{c}, \mathrm{d}$ shows the thermal camera images of the fabricated device at 0 and $3 \mathrm{~V}$, respectively. At 0 $\mathrm{V}$, the temperature profile of the background (author's hand) can be seen through the device. However, at $3 \mathrm{~V}$ the emissivity of the device is significantly suppressed, which screens the background temperature profile (Movie S1). The emissivity of the device can be switched between high and low states many times with a response time of $<1$ s. These devices are thin, light, and flexible and could easily wrap around everyday objects (Figures S1 and S2).

To quantify the performance of the fabricated active thermal surfaces, we first placed them on a hot plate at $55{ }^{\circ} \mathrm{C}$ and recorded the thermal images (Figure 2a,b and Figure $S 1$ and Movie 2) at different bias voltages between 0 and $4 \mathrm{~V}$. Note that the voltage range is limited by the electrochemical window of the room temperature ionic liquid. ${ }^{38} \mathrm{We}$ obtained the best performance with the IL [DEME][TFSI], which yields relatively large electrochemical window up to $4 \mathrm{~V}$. The thermograms show substantial variation in the thermal appearance, which is quite homogeneous over a large area device $\left(10 \times 9 \mathrm{~cm}^{2}\right)$. The IR camera renders the thermograms assuming a constant emissivity of 1 . Although the temperature of the device is the same, the gold electrode appears cold at high voltages due to its low emissivity.

First, we measured the IR spectrum of the emitted radiation at different bias voltages (Figure 2c) using a Fourier transform infrared spectrometer (FTIR). The modulation of spectral radiance of the device covers the full mid-infrared range. The intensity of the spectrum decreases by a factor of 2.5 at $3.5 \mathrm{~V}$ over a broad range. To measure the variation of the total emitted thermal power from the device, we used a thermopile sensor, which performs a differential measurement with respect to the room temperature (inset in Figure 2d). We recorded the output voltage of the sensor as we scanned the bias voltage between 0 and $4 \mathrm{~V}$ with a scan rate of $0.01 \mathrm{~V} / \mathrm{s}$ (Supplementary Movie 2). To block the background radiation, we used a 3 in. silicon wafer coated with $100 \mathrm{~nm}$ thick gold 

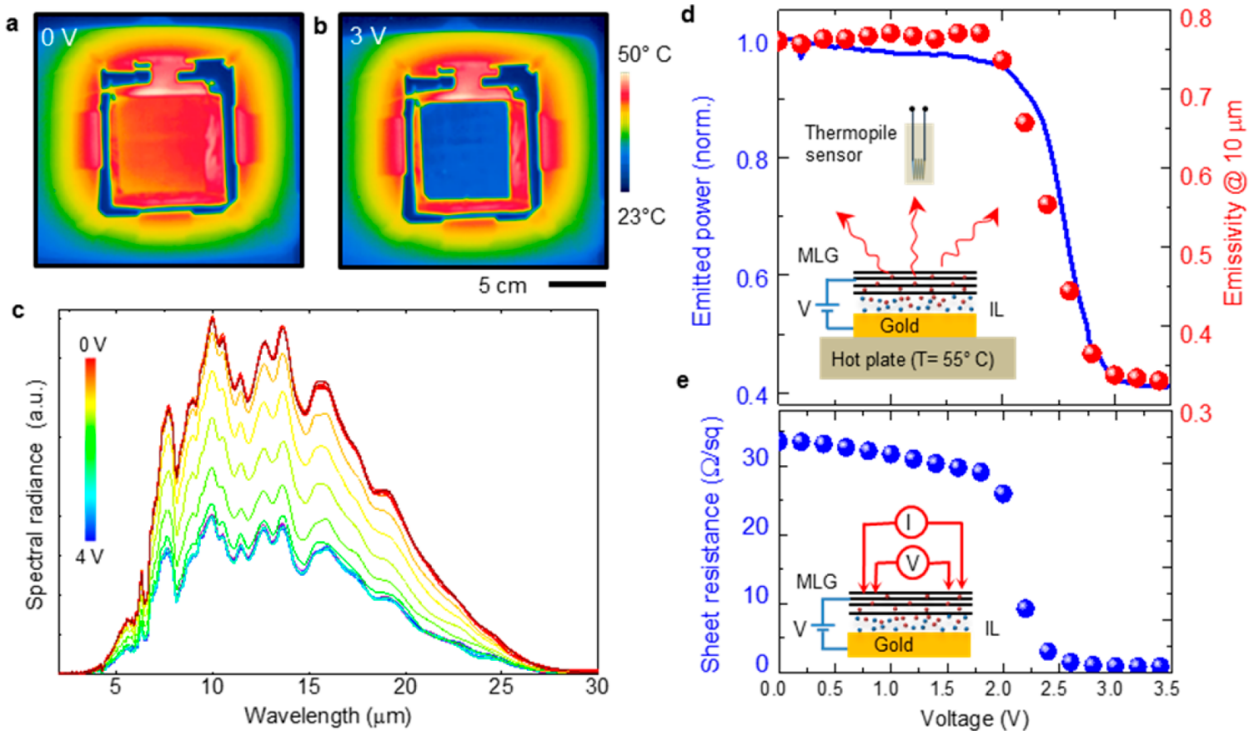

Figure 2. Voltage-controlled thermal emission. (a,b) Thermal camera images of the fabricated device biased at 0 to $3 \mathrm{~V}$, respectively. The device is placed on a hot plate and kept at a temperature of $55^{\circ} \mathrm{C}$. (c) Spectra of the thermal radiation from the device at different bias voltages. (d) Voltage dependence of the emitted thermal power (blue line) and extracted emissivity (red scattered data) at the wavelength of $10 \mu \mathrm{m}$. The thermopile radiation sensor is placed $1 \mathrm{~cm}$ away from the device sitting on a hot plate. The emissivity is calculated using the carbon nanotube forest as a reference. The inset shows the experimental setup used for measuring the voltage dependence of thermal radiation. (e) The sheet resistance of the multilayer graphene electrode plotted against the bias voltage. The inset shows the four-point measurement setup.

film having very low emissivity $(<0.1)$. The voltage dependence of emitted power from the device is shown in Figure $2 \mathrm{~d}$. We observed a clear steplike behavior with a threshold voltage of $2 \mathrm{~V}$. The emitted thermal power is reduced by a factor of 2.5 at a bias voltage of $3.5 \mathrm{~V}$. These numbers agree very well with the spectral measurements.

To calculate the emissivity of the device, we used a carbon nanotube forest as a reference black surface having emissivity close to 1 (Figure S3). ${ }^{39}$ The extracted emissivity of the multilayer graphene at $10 \mu \mathrm{m}$ is reduced from 0.76 down to 0.33 as we scanned the voltage from 0 to $3.5 \mathrm{~V}$ (scattered plot in Figure $2 \mathrm{~d}$ ). Variation of the total radiated power and the extracted emissivity values show similar voltage dependence indicating that the variation of emissivity with the bias voltage is nearly constant over the mid-IR range. The intercalation process is reversible and the device can be switched between high and low emissivity values with a time constant of $0.5 \mathrm{~s}$. We observed a small shift in the threshold voltage due to hysteresis in the intercalation process.

Our results suggest that the observed suppression of the emissivity is due to the suppression of IR absorption of multilayer graphene via intercalation of ionic liquid. To further quantify the intercalation process, we measured variation of the sheet resistance of ML-graphene using four-point resistivity method (inset in Figure 2e). Similarly, the sheet resistance of the graphene electrode shows a step like variation from $33 \Omega$ down to $0.6 \Omega$ (Figure 2e). The sheet resistance and the emissivity of ML-graphene are correlated. As the layer number increases, both sheet resistance and emissivity decrease (Figure S4). To gain more insight into the mechanism behind the electrical control of thermal radiation, we performed in situ optical characterization of the MLgraphene electrodes (Figures S5 and S6). We observed that the transmittance of $\mathrm{ML}$-graphene decreases substantially whereas the reflectivity increases due to the high level of doping. We also tested similar devices with single-layer graphene and observed slight modulation ( $<2 \%$ increase) of thermal radiation due to enhanced interband absorption (Figure S7). These results and our electromagnetic simulations reveal that both interband and intraband transitions of the ML-graphene contribute to the observed emissivity modulation in the IR spectrum $^{35,40,41}$ (Figure S8). The tunable high mobility free carriers on graphene layers are responsible for the control of the emissivity. ${ }^{42,43}$

Using the nonvolatile RTIL electrolyte allows us to operate these devices also in ultrahigh vacuum conditions. This ability is critical for some special applications such as active thermal shields for outer space applications, ${ }^{23}$ as well as utilization of surface characterization tools such as X-ray photoelectron spectroscopy (XPS), which can elucidate the operation of the devices in a chemically specific fashion. Although, intercalation of graphitic materials with metallic ions has been extensively studied, ${ }^{40}$ intercalation of ionic liquids remains relatively unexplored. ${ }^{38}$ Our device layout (Figure 3a) provides a unique advantage to characterize the intercalation process. The ionic liquid contains two nitrogen atoms (Figure $3 b$ ), one with a positive charge (quaternized nitrogen) and the other with a negative charge (imide nitrogen), which yield two wellresolved $\mathrm{N}$ 1s peaks. Figure $3 \mathrm{c}$ shows the recorded $\mathrm{C} 1 \mathrm{~s}, \mathrm{~N}$ $1 \mathrm{~s}$, and F 1s region of XPS spectra at different bias voltages. These spectral evolutions provide a wealth of information about the operation of the device. The appearance of $\mathrm{N} 1 \mathrm{~s}$ and $\mathrm{F} 1 \mathrm{~s}$ peaks after $1.5 \mathrm{~V}$ indicates the onset of the intercalation process and the threshold voltage. Because XPS probes the very top surface $(\sim 10 \mathrm{~nm})$, the appearance of $F 1$ s and $N$ 1s peaks shows that the ions can efficiently intercalate the thick active surface (>100 graphene layers). The intensity of $\mathrm{C} 1 \mathrm{~s}$ decreases with increasing voltage owing to the partial coverage of the top surface with the IL. The $\mathrm{C} 1$ s peak of the $\mathrm{CF}_{3}$ group associated with IL also appears after the threshold voltage. Although, the graphene surface is grounded, the binding energy of $\mathrm{C} 1 \mathrm{~s}$ also experiences a small shift with the applied 

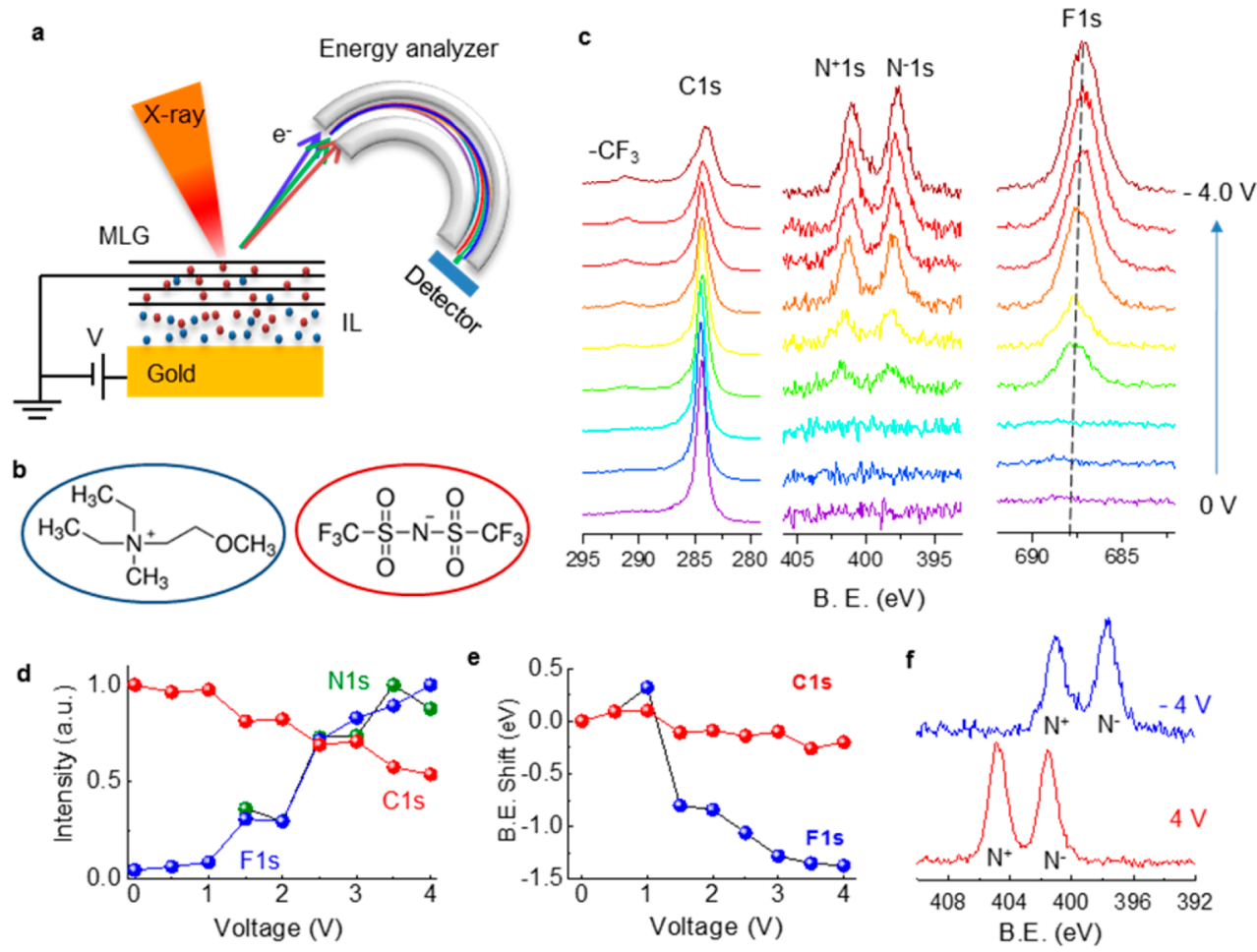

Figure 3. In situ XPS characterization of the active thermal surfaces. (a) Experimental setup used for the operando-XPS. (b) Chemical structure of ionic liquids. Positively and negatively charged nitrogen ions enable monitoring of the chemical content of intercalates. (c) XPS spectra recorded from the surface of device under bias voltages between 0 to $4 \mathrm{~V}$. The spectra were recorded in ultrahigh vacuum $10^{-8}$ Torr. (d) Variation of the normalized intensities and binding energy of $\mathrm{C} 1 \mathrm{~s}, \mathrm{~N}$ 1s, and $\mathrm{F} 1 \mathrm{~s}$. (e) The variation of the binding energy of $\mathrm{C} 1 \mathrm{~s}$ and $\mathrm{F} 1 \mathrm{~s}$. (f) XPS spectra of $\mathrm{N} 1 \mathrm{~s}$ showing the charge imbalance for positive and negative bias voltages.

bias from 284.37 to $283.67 \mathrm{eV}$ (Figure 3e) most likely due to the shift in the Fermi energy of graphene. ${ }^{44}$ Interestingly, we observed cointercalation of anions and cations of the ionic liquid with a significant charge imbalance $>20 \%$ (the ratio of $\mathrm{N}^{-}$to $\mathrm{N}^{+}$). This charge imbalance (due to mobile and quasiindependent ions) is responsible for electrostatic doping on graphene layers. When we apply negative bias voltage, the charge imbalance is reversed (Figure $3 \mathrm{~d}$ ). Our results show that intercalation of ionic liquid into multilayer graphene yields effectively a charge imbalance with a charge excess of about 1 ion for $\sim 200 \mathrm{C}$ atoms of the intercalated active layer (Figure S9). This direct observation of the chemical contents of intercalate with related electronic properties of the graphene layers will further guide us to optimize the device operation.

To show one promising application of the developed thermal surfaces, we now would like to demonstrate a functional adaptive camouflage system. In nature, animals developed adaptive camouflage techniques using specialized cells that enable active feedback mechanisms to adjust the skin color and texture. ${ }^{1,45}$ Our strategy uses thermal emission as a feedback. Figure $4 \mathrm{a}$ shows the working principle of the adaptive thermal camouflage system. The body temperature of the device is set to $40{ }^{\circ} \mathrm{C}$. The thermocouple measures the actual surface temperature of the background and sends the sensory information to the circuit, which uses the thermal radiation from the device as a feedback and yields a control signal to adjust the thermal radiation. The algorithm minimizes the difference between the surface temperature and the apparent temperature of the device. Although the body temperature of the device is constant, by tuning the emissivity of the surface with the control voltage, this device can blend itself with the time varying thermal background. Figure $4 \mathrm{~b}$ shows the varying surface temperature (red curve) and apparent temperature of the device (blue curve). After the optimization of the feedback gain, the apparent temperature follows the surface temperature with a small time delay of $<5 \mathrm{~s}$ (Figure S10). When we set a large gain in the control circuit, we observe large oscillations in the apparent temperature but eventually the apparent temperate reaches that of the background (Figure S11). It is noteworthy that this device can operate in the temperature range between 38 and $25{ }^{\circ} \mathrm{C}$.

The dynamic range of the camouflage system depends on many factors, such as the body temperature of the device, modulation of the emissivity, the surface temperature, the background temperature (from the environment), and quality of thermal contact between the object and the active surface. To obtain more insight into the operation range and further quantify the experimental observations, we developed a quantitative model for the apparent temperature. The thermal camera renders the temperature of a surface from the detected radiation, which includes two parts, (1) the radiation from the surface and, (2) the reflected environment radiation as $\varepsilon_{\mathrm{c}} T_{\mathrm{a}}^{4}=$ $\varepsilon_{0} T_{0}{ }^{4}+R_{0} T_{\mathrm{b}}{ }^{4}$ where $T_{\mathrm{a}}, T_{0}$, and $T_{\mathrm{b}}$ represent apparent, body, and background temperatures, respectively. $\varepsilon_{0}$ is the emissivity of the surface, and $\varepsilon_{\mathrm{c}}$ is the emissivity used by the camera. We can write reflectivity of the surface as $R=1-A=1-\varepsilon_{0}$ where $A$ is the absorption of the surface. Note that the transmission of the device is 0 due to the gold electrode. The solid lines in Figure $4 \mathrm{c}$ shows the relation between apparent temperature and the actual body temperature for different emissivity range from 0 to 1 . For this calculation, we used background temperature of $26.7^{\circ} \mathrm{C}$. We first verify these calculations using 

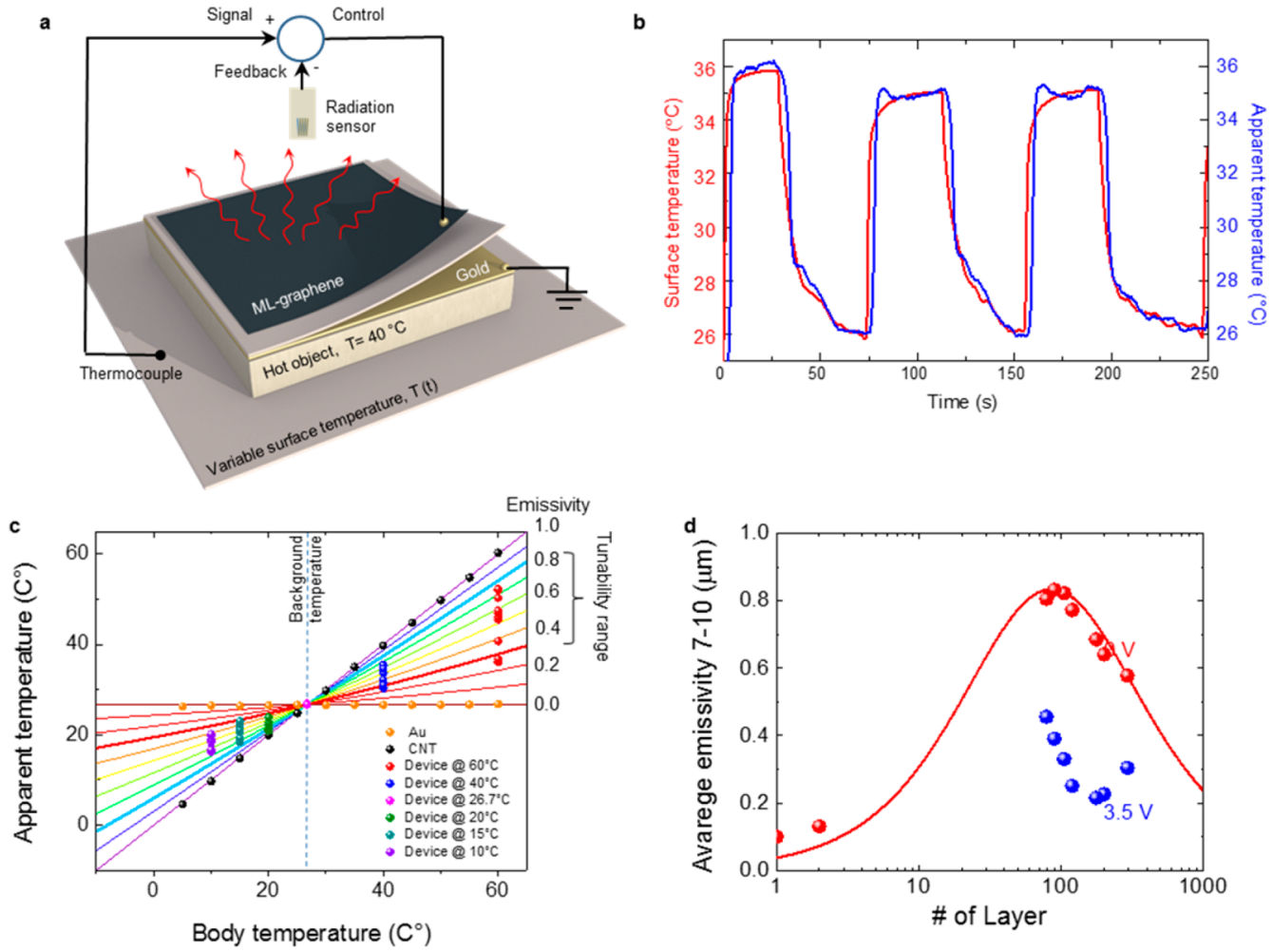

Figure 4. Adaptive thermal camouflage systems. (a) Schematic drawing of the device capable of blending its thermal appearance into a variable temperature background. (b) Time trace of the surface temperature and the apparent temperature of the device. (c) Apparent temperature of a surface plotted against the actual body temperature with different emissivity. The lines show the calculations and the scattered dots represent the measured data. (d) Layer dependence of the averaged emissivity of multilayer graphene (between 7 and $14 \mu \mathrm{m}$ wavelengths for the device configuration given in Figure 1a. The maximum emissivity of 0.8 is obtained around 100 layers. The scattered plot shows the measured values at 0 and $3.5 \mathrm{~V}$ bias voltages.

a gold-coated surface $\left(\varepsilon_{\mathrm{Au}} \sim 0\right)$ and carbon-nanotube sample $\left(\varepsilon_{\mathrm{CNT}} \sim 1\right)$. Gold-coated surface always shows the background temperature due to the perfect IR reflectivity, however, CNT sample shows the actual body temperature due to perfect emissivity (no reflectivity, see Figure S12). Apparent temperature of our device varies between these values depending on the emissivity $(\varepsilon \sim 0.3-0.8)$ and body temperature. Figure $4 c$ reveals three intriguing results due to the interplay between the radiation and reflection. First, the dynamic range of the active surface increases with the temperature difference between the body and the background. Second, when the body temperature is the same with background, the apparent temperature of the device does not change with the applied voltage. The suppression of the emissivity is compensated by the increasing reflectivity. Third, when the body temperature is lower than the background, the apparent temperature increases with decrease in emissivity (increasing voltage). When the voltage is applied, the cold surface looks hotter. Therefore, the voltagecontrolled emissivity and reflectivity of $\mathrm{ML}$-graphene enables us to design new camouflage systems that can disguise not only hot surfaces as cold and but also cold ones as hot in a thermal imaging system. When the surface is hotter than the background temperature, the thermal emission is dominant. Suppression of the emissivity of the surface yields colder appearance. However, when the object is colder than the background temperature, the reflection of the background radiation is dominant. Increasing concentration of high mobility carrier on the graphene surface under a bias voltage yields hotter appearance in thermal imaging systems.
The thickness of the multilayer graphene is another important parameter that defines the modulation range of the emissivity. We fabricated and characterized a series of devices with varying the thickness of the active graphene layer. Figure $4 \mathrm{~d}$ shows the variation of the measured and calculated emissivity with the layer number. The maximum emissivity of 0.8 can be obtained with 100 layers of graphene. Thicker or thinner films yield less emissivity due to larger reflectivity or smaller absorption, respectively. In Figure 4d, we also show the measured emissivity for the doped graphene (at $3.5 \mathrm{~V}$, blue dots). We observed that minimum emissivity also varies with the layer number, which is likely due to inefficient intercalation for thick films and residual infrared absorption of doped graphene in Pauli blocking regime, which is not fully understood yet. The maximum emissivity modulation can be obtained with around 150 layers of graphene.

Finally, we would like to demonstrate an integration scheme which yields more complex reconfigurable thermal images. Figure 5a shows the multipixel device consisting of large area continuous graphene film on PE substrate and $5 \times 5$ arrays of individually addressable gold electrodes deposited on a printed circuit board. In this layout, the graphene film is wired to the ground electrode. The top IR transparent PE layer prevents scratches to the multilayer graphene as well. By controlling the voltage of a pixel with an external circuit, we were able to confine the intercalation within the pixel and thus results in modulation of local emissivity. Figure $5 b$ shows three thermal images of the device with different voltage configurations. For low and high emissivity, we applied -3.5 and $0 \mathrm{~V}$ to the pixels, respectively. A temperature contrast of $10{ }^{\circ} \mathrm{C}$ can be obtained 


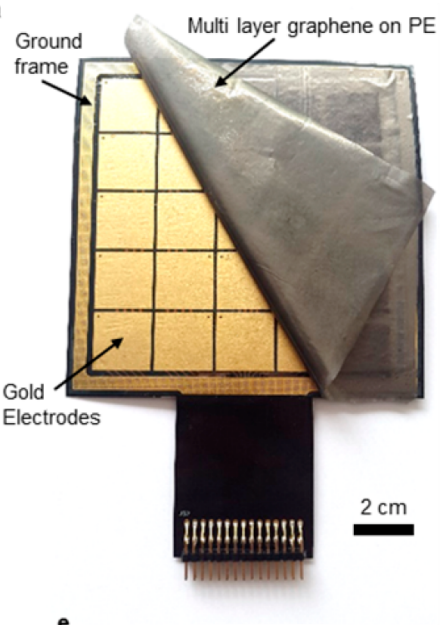

b

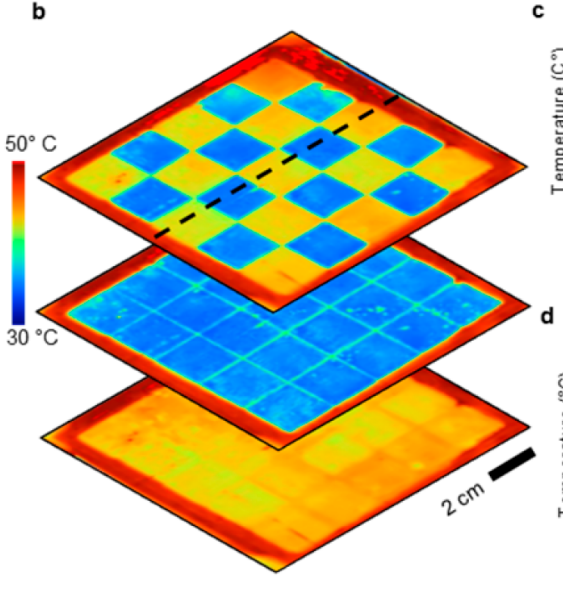

c

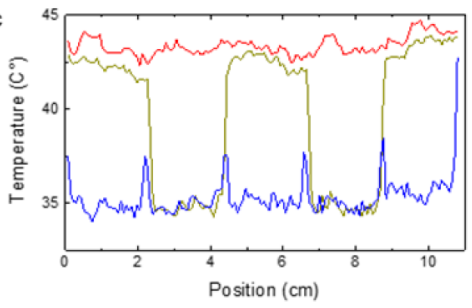

.

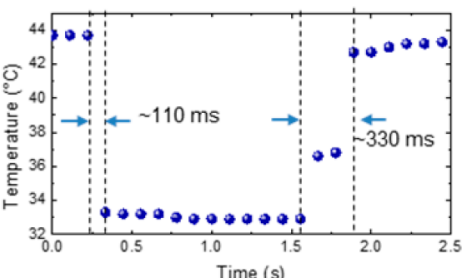

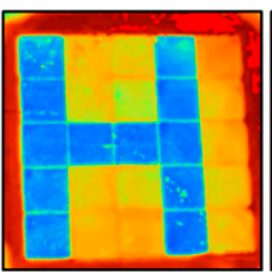
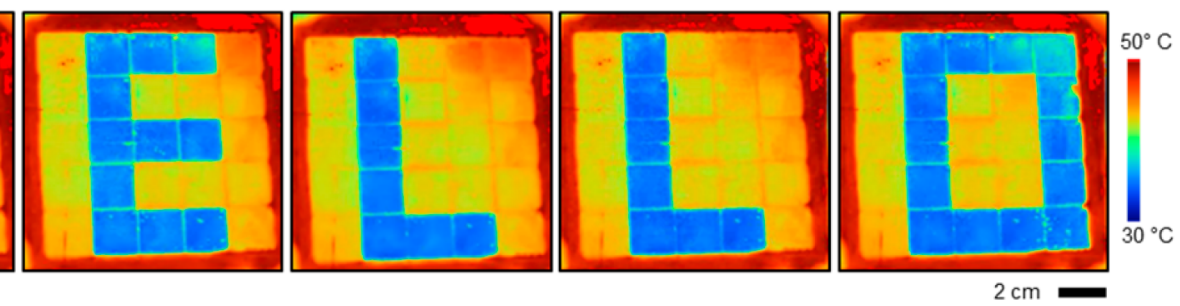

Figure 5. Multipixel active thermal surface. (a) Photograph of the device consisting of $5 \times 5$ arrays of individually addressable pixels with an area of $2 \times 2 \mathrm{~cm}^{2}$. The pixels are defined by the patterned gold electrodes on a printed circuit board and the top graphene layer is wired to the ground electrode. (b) Thermal camera images of the device (heated to $55^{\circ} \mathrm{C}$ ) for three different voltage configurations; all pixels are grounded (bottom), all pixels are biased to $-3.5 \mathrm{~V}$ (middle), and pixels are biased alternatively between 0 and $-3.5 \mathrm{~V}$. (c) Line profile of the apparent temperature of the device shown in (b). (d) Time-trace of the apparent temperature of the device switched between different voltage configurations. (e) Complex thermal images of text "HELLO" generated by the device.

at each pixel individually (Figure 5c) and can be switched in $0.1 \mathrm{~s}$ (Figure 5d). The crosstalk between the pixels is negligible. With this area-selective intercalation, we generated complex thermal images such as a text "HELLO" (see Movie 4). The size of the pixels can be scaled down to millimeter without a significant crosstalk. These devices can also be fabricated by patterning the graphene layer and using different addressing mechanisms (see Figures S13 and S14). These results show that our approach can be used to disguise the shape and temperature of objects in thermal imaging systems. Furthermore, these devices can also operate as adaptive IRmirrors. These devices can operate up to 500 full operation cycles in ambient conditions. However, we observed degradation in the device performance likely due to hydration of the ionic liquid in ambient conditions and corrosion of the gold electrode. We believe that reliability of the device can be improved significantly by a passivation of the device.

In conclusion, we have developed a new class of active thermal surfaces capable of efficient real-time electrical-control of their thermal emission over the full infrared spectrum. We showed that emissivity of multilayer graphene electrodes can be controlled electrically between 0.8 down to 0.3 with a bias voltage less than $4 \mathrm{~V}$. Using these active surfaces, we have demonstrated adaptive camouflage systems that can disguise hot surfaces as cold and cold ones as hot in a thermal imaging system. Simplicity of the layered device structure together with the efficient modulation over broad IR spectrum (from 2 to 25 $\mu \mathrm{m})$ provides an unprecedented ability for adaptive thermal camouflage. These active surfaces are flexible which enable their integration with nonplanar surfaces, such as soft robotic systems. ${ }^{2}$ Fabricating these devices on strained elastomers could provide possibilities for stretchable camouflage devices. Furthermore, these devices can operate at high temperatures and under high vacuum conditions due to low vapor pressure of the ionic liquids enabling us to monitor the intercalation process using X-ray photoelectron spectroscopy. Our results provide a significant step for realization of adaptive thermal management, which could enable new technologies, not only for thermal camouflage but also for adaptive IR optics and adaptive heat shields for satellites. ${ }^{23}$

Methods. Synthesis and Transfer Printing of Multilayer Graphene. We synthesized multilayer graphene on $50 \mu \mathrm{m}$ thick Ni foil substrates (Alfa Aesar Item \#12722) using a chemical vapor deposition system. By adjusting the growth temperature between 900 to $1050{ }^{\circ} \mathrm{C}$, we controlled the number of graphene layers from 60 to 100 layers. During the growth, we used $30 \mathrm{sccm}$ of $\mathrm{CH}_{4}$ and $100 \mathrm{sccm} \mathrm{Ar}$ and 100 $\mathrm{sccm} \mathrm{H}_{2}$ gases at ambient pressure. The growth duration was 5 min. After cooling the samples to room temperature, we etched the $\mathrm{Ni}$ foil in a $\mathrm{FeCl}_{3}$ solution $(1 \mathrm{M})$. We transferred the MLgraphene on a clean water surface. The surface of graphene is hydrophobic allowing free-standing ML-graphene film on the water surface. By immersing the polyethylene membrane into the water, graphene conformably coats the surface.

Fabrication of Active Thermal Surfaces. After the transfer process, we injected room-temperature ionic liquid electrolyte [DEME][TFSI] (98.5\%, diethylmethyl(2-methoxyethyl)ammoniumbis(trifluoromethylsulfonyl)imide, Sigma-Aldrich, 727679) into the membrane and attached copper wires on the ML-graphene with a conductive tape. To fabricate the gold electrode, we evaporated $5 \mathrm{~nm}$ Ti adhesive layer and 100 $\mathrm{nm}$ Au layer on $25 \mu \mathrm{m}$ thick heat resistive nylon using thermal 
evaporation. We placed the PE membrane on the gold coated nylon.

Thermal Imaging. The thermographs of the samples were recorded using FLIR A40 thermal camera. The camera renders the thermographs using constant emissivity of 1 .

Electrical Measurements. To apply the bias voltage to the devices, we used Keithley 2400 source measure unit. We recorded both voltage and charging current during the intercalation and deintercalation processes. To measure sheet resistance, we used 4-point resistance measurement system (Nano Magnetics Inc.), which includes two separate source meters (Keithley 2400 and 2600). The first power supply applies the bias voltage between the $\mathrm{ML}$-graphene and the gold electrodes to initiate intercalation and the second one measures the sheet resistance.

Spectroscopic Characterization. Thermal emission measurements were performed using Bruker Vertex 70v Fourier transform infrared spectrometer (FTIR). The devices were placed on a hot plate at constant temperature of $55{ }^{\circ} \mathrm{C}$. The hot plate is aligned to the emission port of the spectrometer. We used wide range DLATGS detector (D201/BD) and widerange beam splitter (T240) in the spectrometer. A Thermo Fisher K-Alpha spectrometer was used for XPS characterizations.

\section{ASSOCIATED CONTENT}

\section{S Supporting Information}

The Supporting Information is available free of charge on the ACS Publications website at DOI: 10.1021/acs.nanolett.8b01746.

Additional details on the experimental setup, characterization, and analysis of multilayer graphene films, characterization of control devices, detailed theoretical analysis, additional experimental results, and comparison of electrochromic materials used for IR emissivity control (PDF)

Real time thermal movie of an operating device placed on the author's hand (AVI)

Real time thermal movie of a large area device placed on a hot plate (AVI)

Real time thermal movie of an operating device placed on a hot plate under linear voltage sweep (AVI)

Real time thermal movie of the multipixel active thermal surface (AVI)

\section{AUTHOR INFORMATION}

\section{Corresponding Author}

*E-mail: coskun.kocabas@manchester.ac.uk.

\section{ORCID $\odot$}

Nurbek Kakenov: 0000-0003-2321-6157

Sinan Balci: 0000-0002-9809-8688

Sefik Süzer: 0000-0002-5866-2600

Coskun Kocabas: 0000-0003-0831-5552

\section{Author Contributions}

C.K. and O.S. proposed the idea and planned the experiments. B.U synthesized the samples. B.U and O.S. fabricated the devices. B.U., O.Y., O.S., and C.K. performed the experiments. S.S. performed the XPS measurements and analyzed the data. S.B., S.A., N.K., O.B., and O.S. helped for the measurements and electromagnetic modeling of the devices. S.O. designed the multipixel device. C.K. analyzed the data and wrote the manuscript. All authors discussed the results and contributed to the scientific interpretation as well as to the writing of the manuscript.

\section{Notes}

The authors declare no competing financial interest.

\section{ACKNOWLEDGMENTS}

C.K. acknowledges the financial support from European Research Counsel for ERC- Consolidator Grant SmartGraphene 682723. C.K. acknowledges BAGEP Award of the Science Academy.

\section{REFERENCES}

(1) Ramachandran, V. S.; Tyler, C. W.; Gregory, R. L.; RogersRamachandran, D.; Duensing, S.; Pillsbury, C.; Ramachandran, C. Nature 1996, 379 (6568), 815-818.

(2) Morin, S. A.; Shepherd, R. F.; Kwok, S. W.; Stokes, A. A.; Nemiroski, A.; Whitesides, G. M. Science 2012, 337 (6096), 828-832.

(3) Schittny, R.; Kadic, M.; Guenneau, S.; Wegener, M. Phys. Rev. Lett. 2013, 110 (19), 195901.

(4) Han, T. C.; Bai, X.; Gao, D. L.; Thong, J. T. L.; Li, B. W.; Qiu, C. W. Phys. Rev. Lett. 2014, 112 (5), 054302.

(5) Han, T. C.; Bai, X.; Thong, J. T. L.; Li, B. W.; Qiu, C. W. Adv. Mater. 2014, 26 (11), 1731-1734.

(6) Yu, C. J.; Li, Y. H.; Zhang, X.; Huang, X.; Malyarchuk, V.; Wang, S. D.; Shi, Y.; Gao, L.; Su, Y. W.; Zhang, Y. H.; Xu, H. X.; Hanlon, R. T.; Huang, Y. G.; Rogers, J. A. Proc. Natl. Acad. Sci. U. S. A. 2014, 111 (36), 12998-13003.

(7) Raman, A. P.; Anoma, M. A.; Zhu, L.; Rephaeli, E.; Fan, S. Nature 2014, 515 (7528), 540-4.

(8) Lampert, C. M. Sol. Energy Mater. 1984, 11 (1-2), 1-27.

(9) Luo, C. Y.; Narayanaswamy, A.; Chen, G.; Joannopoulos, J. D. Phys. Rev. Lett. 2004, 93 (21), 213905.

(10) Laroche, M.; Carminati, R.; Greffet, J. J. Phys. Rev. Lett. 2006, 96 (12), 123903.

(11) Han, S. E.; Norris, D. J. Phys. Rev. Lett. 2010, 104 (4), 043901.

(12) Tsai, M. W.; Chuang, T. H.; Meng, C. Y.; Chang, Y. T.; Lee, S. C. Appl. Phys. Lett. 2006, 89 (17), 251102.

(13) Kats, M. A.; Blanchard, R.; Zhang, S. Y.; Genevet, P.; Ko, C. H.; Ramanathan, S.; Capasso, F. Phys. Rev. X 2013, 3 (4), 041004.

(14) Bessiere, A.; Marcel, C.; Morcrette, M.; Tarascon, J. M.; Lucas, V.; Viana, B.; Baffier, N. J. Appl. Phys. 2002, 91 (3), 1589-1594.

(15) Sauvet, K.; Sauques, L.; Rougier, A. Sol. Energy Mater. Sol. Cells 2009, 93 (12), 2045-2049.

(16) Xiao, L.; Ma, H.; Liu, J. K.; Zhao, W.; Jia, Y.; Zhao, Q.; Liu, K.; Wu, Y.; Wei, Y.; Fan, S. S.; Jiang, K. L. Nano Lett. 2015, 15 (12), $8365-8370$

(17) Inoue, T.; De Zoysa, M.; Asano, T.; Noda, S. Nat. Mater. 2014, 13 (10), 928-31.

(18) Hutchins, M. G.; Butt, N. S.; Topping, A. J.; Gallego, J.; Milne, P.; Jeffrey, D.; Brotherston, I. Electrochim. Acta 2001, 46 (13-14), 1983-1988.

(19) Huang, Y.; Boriskina, S. V.; Chen, G. Appl. Phys. Lett. 2014, 105 (24), 244102.

(20) Vassant, S.; Doyen, I. M.; Marquier, F.; Pardo, F.; Gennser, U.; Cavanna, A.; Pelouard, J. L.; Greffet, J. J. Appl. Phys. Lett. 2013, 102 (8), 051127.

(21) Schuller, J. A.; Taubner, T.; Brongersma, M. L. Nat. Photonics 2009, 3 (11), 658-661.

(22) Jun, Y. C.; Luk, T. S.; Ellis, A. R.; Klem, J. F.; Brener, I. Appl. Phys. Lett. 2014, 105 (13), 131109.

(23) Demiryont, H.; Moorehead, D. Sol. Energy Mater. Sol. Cells 2009, 93 (12), 2075-2078.

(24) Li, H.; Xie, K.; Pan, Y.; Yao, M.; Xin, C. Synth. Met. 2009, 159 (13), 1386-1388.

(25) Mortimer, R. J. Annu. Rev. Mater. Res. 2011, 41, 241-268. 
(26) Sensale-Rodriguez, B.; Yan, R.; Kelly, M. M.; Fang, T.; Tahy, K.; Hwang, W. S.; Jena, D.; Liu, L.; Xing, H. G. Nat. Commun. 2012, 3, 780-786.

(27) Liu, M.; Yin, X. B.; Ulin-Avila, E.; Geng, B. S.; Zentgraf, T.; Ju, L.; Wang, F.; et al. Nature 2011, 474 (7349), 64-67.

(28) Balci, O.; Polat, E. O.; Kakenov, N.; Kocabas, C. Nat. Commun. 2015, 6, 6628.

(29) Shi, C.; Mahlmeister, N. H.; Luxmoore, I. J.; Nash, G. R. Nano Res. 2017, 10, 1-7.

(30) Barnard, H. R.; Zossimova, E.; Mahlmeister, N. H.; Lawton, L. M.; Luxmoore, I. J.; Nash, G. R. Appl. Phys. Lett. 2016, 108 (13), 131110.

(31) Mahlmeister, N. H.; Lawton, L. M.; Luxmoore, I. J.; Nash, G. R. Appl. Phys. Express 2016, 9 (1), 012105.

(32) Balci, O.; Kakenov, N.; Kocabas, C. Appl. Phys. Lett. 2017, 110 (16), 161102.

(33) Balci, O.; Kakenov, N.; Karademir, E.; Balci, S.; Cakmakyapan, S.; Polat, E. O.; Caglayan, H.; Ozbay, E.; Kocabas, C. Science Advances 2018, 4 (1), eaao1749.

(34) Polat, E. O.; Kocabas, C. Nano Lett. 2013, 13 (12), 58515857.

(35) Bao, W. Z.; Wan, J. Y.; Han, X. G.; Cai, X. H.; Zhu, H. L.; Kim, D. H.; Ma, D. K.; Xu, Y. L.; Munday, J. N.; Drew, H. D.; Fuhrer, M. S.; Hu, L. B. Nat. Commun. 2014, 5, 4224.

(36) Brar, V. W.; Sherrott, M. C.; Jang, M. S.; Kim, S.; Kim, L.; Choi, M.; Sweatlock, L. A.; Atwater, H. A. Nat. Commun. 2015, 6, 7032.

(37) Polat, E. O.; Balci, O.; Kocabas, C. Sci. Rep. 2015, 4, 6484.

(38) Armand, M.; Endres, F.; MacFarlane, D. R.; Ohno, H.; Scrosati, B. Nat. Mater. 2009, 8 (8), 621-629.

(39) Mizuno, K.; Ishii, J.; Kishida, H.; Hayamizu, Y.; Yasuda, S.; Futaba, D. N.; Yumura, M.; Hata, K. Proc. Natl. Acad. Sci. U. S. A. 2009, 106 (15), 6044-6047.

(40) Dresselhaus, M.; Dresselhaus, G. Adv. Phys. 1981, 30 (2), 139326.

(41) Hennig, G. J. Chem. Phys. 1965, 43 (4), 1201-1206.

(42) Solanki, A. K.; Kashyap, A.; Nautiyal, T.; Auluck, S.; Khan, M. A. Solid State Commun. 1996, 100 (9), 645-649.

(43) Taft, E. A.; Philipp, H. R. Phys. Rev. 1965, 138 (1A), A197.

(44) Copuroglu, M.; Aydogan, P.; Polat, E. O.; Kocabas, C.; Suzer, S. Nano Lett. 2014, 14 (5), 2837-2842.

(45) Kreit, E.; Mathger, L. M.; Hanlon, R. T.; Dennis, P. B.; Naik, R. R.; Forsythe, E.; Heikenfeld, J. J. R. Soc., Interface 2013, 10 (78), 20120601. 